\title{
Evaluation of water properties in HEA- HEMA hydrogels swollen in aqueous-PEG solutions using thermoanalytical techniques
}

Article

Accepted Version

Hackl, E. V., Khutoryanskiy, V. V., Tiguman, G. M. B. and Ermolina, I. (2015) Evaluation of water properties in HEAHEMA hydrogels swollen in aqueous-PEG solutions using thermoanalytical techniques. Journal of Thermal Analysis and Calorimetry, 121 (1). pp. 335-345. ISSN 1588-2926 doi: https://doi.org/10.1007/s10973-015-4446-y Available at https://centaur.reading.ac.uk/40314/

It is advisable to refer to the publisher's version if you intend to cite from the work. See Guidance on citing.

Published version at: http://link.springer.com/article/10.1007/s10973-015-4446-y

To link to this article DOI: http://dx.doi.org/10.1007/s10973-015-4446-y

Publisher: Springer Netherlands

All outputs in CentAUR are protected by Intellectual Property Rights law, including copyright law. Copyright and IPR is retained by the creators or other copyright holders. Terms and conditions for use of this material are defined in the End User Agreement. 


\section{www.reading.ac.uk/centaur}

\section{CentAUR}

Central Archive at the University of Reading

Reading's research outputs online 


\section{Journal of Thermal Analysis and Calorimetry \\ Evaluation of water properties in HEA-HEMA hydrogels swollen in aqueous-PEG solutions using thermoanalytical techniques \\ --Manuscript Draft--}

\begin{tabular}{|c|c|}
\hline \multicolumn{2}{|l|}{ Manuscript Number: } \\
\hline Full Title: & $\begin{array}{l}\text { Evaluation of water properties in HEA-HEMA hydrogels swollen in aqueous-PEG } \\
\text { solutions using thermoanalytical techniques }\end{array}$ \\
\hline Article Type: & Original Research \\
\hline Corresponding Author: & $\begin{array}{l}\text { Irina Ermolina, Ph.D. } \\
\text { De Montfort University } \\
\text { Leicester, UNITED KINGDOM }\end{array}$ \\
\hline \multicolumn{2}{|l|}{$\begin{array}{l}\text { Corresponding Author Secondary } \\
\text { Information: }\end{array}$} \\
\hline Corresponding Author's Institution: & De Montfort University \\
\hline \multicolumn{2}{|l|}{$\begin{array}{l}\text { Corresponding Author's Secondary } \\
\text { Institution: }\end{array}$} \\
\hline First Author: & Ellen V. Hackl, PhD \\
\hline \multicolumn{2}{|l|}{ First Author Secondary Information: } \\
\hline \multirow[t]{4}{*}{ Order of Authors: } & Ellen V. Hackl, PhD \\
\hline & Vitaliy V. Khutoryanskiy, PhD \\
\hline & Gustavo M.B. Tiguman \\
\hline & Irina Ermolina, Ph.D. \\
\hline \multicolumn{2}{|c|}{ Order of Authors Secondary Information: } \\
\hline Abstract: & $\begin{array}{l}\text { Hydrogels are polymeric materials used in many pharmaceutical and biomedical } \\
\text { applications due to their ability to form the 3-D hydrophilic polymeric networks, which } \\
\text { can absorb large amounts of water. In the present work polyethylene glycols (PEG) } \\
\text { were introduced into the hydrogel liquid phase in order to improve the mechanical } \\
\text { properties of hydrogels composed of 2-hydroxyethylacrylate and 2- } \\
\text { hydroxyethylmethacrylate (HEA-HEMA) synthesized with different co-monomer } \\
\text { compositions and equilibrated in water or in } 20 \% \text { water-PEG } 400 \text { Da and } 600 \mathrm{Da} \\
\text { solutions. The thermoanalytical techniques (differential scanning calorimetry (DSC) } \\
\text { and thermogravimetry (TG)) were used to evaluate the amount and properties of free } \\
\text { and bound water in HEA-HEMA hydrogels. Internal structure and the mechanical } \\
\text { properties of hydrogels were studied using scanning electron microscopy and friability } \\
\text { assay. TG 'loss-on-drying' experiments were applied to study the water-retention } \\
\text { properties of hydrogels whereas the combination of TG and DSC allowed estimating } \\
\text { the total amount of freezable and non-freezing water in hydrogels. } \\
\text { The results show that the addition of the viscous co-solvent (PEG) to the liquid medium } \\
\text { results in significant improvement of the mechanical properties of HEA-HEMA } \\
\text { hydrogels and also slightly retards the water loss from hydrogels. A redistribution of } \\
\text { free and bound water in the hydrogels equilibrated in mixed solutions containing } 20 \\
\text { vol\% of PEGs takes place. }\end{array}$ \\
\hline \multirow[t]{2}{*}{ Suggested Reviewers: } & $\begin{array}{l}\text { Polycarpos Pissis } \\
\text { National Technical University of Athens } \\
\text { ppissis@central.ntua.gr }\end{array}$ \\
\hline & $\begin{array}{l}\text { Jacek Ulanski } \\
\text { Professor, Technical University of Lodz } \\
\text { julanski@p.lodz.pl }\end{array}$ \\
\hline
\end{tabular}


1

2

Ellen V. Hackl ${ }^{1}$, Vitaliy V. Khutoryanskiy ${ }^{2}$, Gustavo M.B. Tiguman ${ }^{1,3}$, Irina Ermolina ${ }^{1^{*}}$

\title{
Evaluation of water properties in HEA-HEMA hydrogels swollen in aqueous-PEG solutions using thermoanalytical techniques
}

\author{
${ }^{1}$ Leicester School of Pharmacy, De Montfort University, Leicester, UK \\ ${ }^{2}$ Reading School of Pharmacy, University of Reading, Reading, UK \\ ${ }^{3}$ Faculty of Pharmaceutical Sciences, Universidade de Sao Paulo, Brazil \\ * Corresponding author, email: iermolina@dmu.ac.uk
}

Key words: hydrogels, mechanical properties, free water, bound water, DSC, TG

\begin{abstract}
Hydrogels are polymeric materials used in many pharmaceutical and biomedical applications due to their ability to form the 3-D hydrophilic polymeric networks, which can absorb large amounts of water. In the present work polyethylene glycols (PEG) were introduced into the hydrogel liquid phase in order to improve the mechanical properties of hydrogels composed of 2-hydroxyethylacrylate and 2-hydroxyethylmethacrylate (HEA-HEMA) synthesized with different co-monomer compositions and equilibrated in water or in 20\% water-PEG $400 \mathrm{Da}$ and $600 \mathrm{Da}$ solutions. The thermoanalytical techniques (differential scanning calorimetry (DSC) and thermogravimetry (TG)) were used to evaluate the amount and properties of free and bound water in HEA-HEMA hydrogels. Internal structure and the mechanical properties of hydrogels were studied using scanning electron microscopy and friability assay. TG 'loss-on-drying' experiments were applied to study the water-retention properties of hydrogels whereas the combination of TG and DSC allowed estimating the total amount of freezable and non-freezing water in hydrogels.

The results show that the addition of the viscous co-solvent (PEG) to the liquid medium results in significant improvement of the mechanical properties of HEA-HEMA hydrogels and also slightly retards the water loss from hydrogels. A redistribution of free and bound water in the hydrogels equilibrated in mixed solutions containing 20 vol\% of PEGs takes place.
\end{abstract}




\section{Introduction}

Hydrogels are three dimensional hydrophilic polymer networks, which are able to absorb and retain a significant amount of water (from 10-20\% up to thousand of times their dry mass) [1,2,3]. Hydrogels are used in many pharmaceutical and biomedical applications (i.e. drug delivery systems [1,4], contact lenses and ocular drug delivery $[5,6]$, wound dressings, tissue engineering [7, 8], intelligent sensitive sensors [1]) due to their excellent biocompatibility as they have properties similar to living tissues and do not trigger responses or rejections from the immune system. Hydrogels can be classified as chemical (permanent) if they are covalently cross-linked networks, or physical (reversible) if they are composed of non-covalently cross-linked networks and macromolecules are held together by ionic bonds, H-bonding and/or hydrophobic effects [1]. Chemically cross-linked hydrogels are usually mechanically stronger compared to physical gels.

Hydrogels can accommodate substantial amount of water, which has different properties depending on the position and interactions within the hydrophilic network. A "three states model" presented in 1973 by John and Andrade, defines tree types of water in hydrogels - non-freezing or bound water, free or bulk water and freezing interfacial or intermediate water [9]. Molecules of free water are not affected by the polymer and freeze / melt similarly to pure water; molecules of bound water are directly immobilized by binding to the polymer chains through hydrogen bonds. Molecules of intermediate or freezing bound water interact weakly with polymers. Some authors suggest that the polar interaction between water and polymer chains cannot be the sole factor responsible for the existence of nonfreezing water; other factors including increased viscosity at low temperatures and inability for water molecules to diffuse through the hydrogel phase also need to be taken into account [10 and references therein].

It was widely shown that properties of bound water (phase transitions, relaxation time, polarization, etc.) differ from properties of free water. As a result, diffusion of the water-soluble drug molecules in hydrogels is expected to be affected by the presence of different types of water. It is assumed that at high level of hydration, the drug diffusion would occur primarily in the bulk water; however at low hydration, the diffusion can take place in the bound water $[11,12]$. The diffusion in bound water can be reduced due to additional steric hindrance, increased viscous drag, or reduced compatibility with a diffusing solute.

Several atomistic models have been proposed to describe the interstitial diffusion based on the motions of free water. For instance, according to the Mackie and Meares model [13], the polymer blocks a fraction of the sites restricting the solute diffusion to the free sites. In this case the diffusion coefficient D for a solute in the gel depends on the volume fraction of the free water (the porosity fraction), $\varepsilon: f=\frac{D}{D_{0}}=(\varepsilon /(2-\varepsilon))^{2}$, where $\mathrm{D}_{0}$ is the diffusion coefficient in water. To obtain $f<0.1$ (meaning the diffusion time increases 10 times), $\varepsilon$ must be below 0.5 .

The drug uptake/release properties are strongly dependent on the diffusive behavior of the water; therefore the determination of the amount of free and bound water in hydrogels is very important for designing the intelligent drug 
is important for the understanding of the nature of interactions between polymer and water.

Several methods were previously applied to determine the different types of water in hydrogels including NMR [14, $15,16]$, dilatometry, conductivity measurements and thermal analysis [10, 17, 18 and references therein].

Polymer hydrogels (synthetic or natural) can be synthesized as homopolymers or co-polymers. Poly(2-hydroxyethyl methacrylate) (polyHEMA or PHEMA) is one of the most widely used hydrogel materials. The properties of PHEMA hydrogels have been extensively investigated in the number of studies [19 and references therein]. PHEMA has generally been regarded as a hydrophilic, but water insoluble material. It contains both hydrophobic (methyl) and hydrophilic pendant groups $(\mathrm{OH}, \mathrm{CO})$, which are responsible for the hydrogel swelling. In a swollen state it becomes soft and rubber-like thermostable material with a high mechanical strength.

Different properties of hydrogels (such as oxygen permeability, the rate of drug release and release mechanism, etc.) can be adjusted by changing the water content in hydrogels. In order to increase the amount of water accumulated by hydrogels (the hydrogel swelling degree) HEMA monomers can be co-polymerized with more hydrophilic monomers [20, 21, 22, 23 ]. Recently Khutoryanskiy and co-workers [24] synthesized cross-linked HEA-HEMA copolymer hydrogels with different HEA-to-HEMA ratios and studied their swelling and mechanical properties. It was shown that co-polymerisation of HEMA with more hydrophilic HEA can significantly increase the water content in hydrogels (the equilibrium swelling degree) when HEA content in hydrogels increases above $40 \mathrm{~mol} \%$. At the same time hydrogels with large HEA content (above 50-60 mol\%) and significantly enriched water content were shown to have inferior mechanical properties (mechanical weaknesses). Increased friability of hydrogels makes them very difficult to handle, which limits their potential application.

In the present work oligomeric polyethylene glycols (PEG) with molecular weights of 400 and 600 Da were introduced into the liquid phase in order to overcome mechanical weaknesses and to improve the mechanical properties of cross-linked HEA-HEMA hydrogels. Polyethylene glycols with different molecular weights are widely used as excipients in cosmetics and pharmaceutical industry due to excellent solubility in water and outstanding toxicological safety [25]. However, addition of PEG into formulation can have an impact on water content and freebound water redistribution in hydrogels resulting in alteration in drug diffusion and uptake/release properties. Therefore the state of water in HEA-HEMA hydrogels in PEG solutions needs to be examined to establish the PEG impact on the liquid medium and physicochemical properties of the hydrogels. To evaluate the amount and properties of free and bound water in HEA-HEMA hydrogels, in the present work the thermoanalytical techniques (DSC and TG) were applied. Additionally, scanning electron microscopy (SEM) was engaged for the structure analysis of the fully swollen hydrogels. The friability test was applied to characterize the mechanical properties of hydrogels with different compositions and liquid media. 


\section{Materials and Methods}

\section{Materials}

The cross-linked 2-hydroxyethylacrylate-co-2-hydroxyethylmethacrylate (HEA-HEMA) hydrogels with different copolymer compositions were synthesized by three-dimensional free-radical copolymerisation of HEA and HEMA as described previously [24]. Eleven hydrogel samples with different HEA/HEMA ratios (HEA content varied from 0 to $100 \mathrm{~mol} \%$ ) were prepared. All hydrogels are labelled according to the HEA mol\% content in the monomer feed mixture.

PEG with the average molecular weights of 400 and 600 Da were purchased from Fluka.

Freeze-dried hydrogels were immersed in different liquid media including distilled water, 20 vol\% aqueous solutions of PEG 400 and 20 vol\% aqueous solutions of PEG $600 \mathrm{Da}$. Hydrogels were fully swollen in a large excess of the liquid media at $4{ }^{\circ} \mathrm{C}$ until the mass of hydrogel became constant over time.

\section{Methods}

\section{Swelling of HEA-HEMA hydrogels}

Dry hydrogels were immersed into an excessive amount of liquid (distilled water or $20 \mathrm{vol} \%$ aqueous solutions of PEG 400 or PEG 600) at room temperature. The mass of the hydrogels was measured gravimetrically at regular time intervals. Before each measurement surface water was accurately removed by blotting each sample against plastic weighing boat. The experiment was continued until the mass of a sample remained constant. Swelling degree (in \%) at each time was calculated as

$$
S D \%=\frac{W_{s}-W_{d}}{W_{d}} \cdot 100 \%
$$

where $W_{s}$ is mass of a swollen hydrogel; $W_{d}$ is mass of a dry hydrogel.

\section{Thermogravimetry (TG)}

TG measurements of fully-swollen HEA-HEMA hydrogels were carried out using the Perkin Elmer Pyris 1 Thermogravimetric Analyzer (USA). Nitrogen was used as purge gas with the flow rate of $20 \mathrm{~mL} \mathrm{~min}^{-1}$; nitrogen flow conditions were kept constant during all experiments. Two types of experiments were conducted:

(i) Temperature scanning: hydrogel samples $(25-30 \mathrm{mg})$ were embedded into non-hermetic aluminium pans sealed up with a lid perforated by a needle. Samples were heated from room temperature to $250{ }^{\circ} \mathrm{C}$ at a heating rate of $5{ }^{\circ} \mathrm{C}$ $\min ^{-1}$.

(ii) Loss on drying experiments: hydrogel sample of $25-30 \mathrm{mg}$ was placed in uncovered aluminium pan and change in the sample mass was measured at constant temperature $\left(37^{\circ} \mathrm{C}\right)$ and controlled purged gas for 120 minutes. To evaporate water remained after hydrogel isothermal drying at physiological temperature sample was heated from 
$37{ }^{\circ} \mathrm{C}$ to $250{ }^{\circ} \mathrm{C}$ at a rate of $5{ }^{\circ} \mathrm{C} \mathrm{min}{ }^{-1}$. Water evaporated during incubation at $37{ }^{\circ} \mathrm{C}$ and total water in hydrogel samples were calculated.

\section{Differential scanning calorimetry (DSC)}

DSC measurements were performed in a Perkin-Elmer Jade DSC (USA). Swollen hydrogel samples (about 9-12 mg) were crimped in an aluminum pan. An empty aluminum pan was used as a reference. The samples were subjected to a cooling scan from $30{ }^{\circ} \mathrm{C}$ down to $-35^{\circ} \mathrm{C}\left(-45^{\circ} \mathrm{C}\right.$ for hydrogels swollen in $20 \mathrm{vol} \%$ PEG solutions) and equilibrated for $3 \mathrm{~min}$, followed by a heating scan from that temperature up to $30^{\circ} \mathrm{C}$. Both scans were performed at the rate of $2{ }^{\circ} \mathrm{C} \mathrm{min}^{-1}$. Nitrogen was used as a purge gas at the flow rate of $30 \mathrm{~mL} \mathrm{~min}^{-1}$. The calorimeter was calibrated using indium standard.

\section{Friability of fully swollen hydrogels}

Mechanical stability of the hydrogels with different HEA contents in aqueous and in 20 vol\% PEG solutions was studied using an Erweka Roche friability tester (Germany). In a typical experiment a fully swollen hydrogel sample with mass ranging between $25 \mathrm{mg}$ to $40 \mathrm{mg}$ was placed in a plastic spheroidal container of $45.1 \mathrm{~mm}$ long with an internal diameter of $35.1 \mathrm{~mm}$. To increase the damaging force a small plastic bead with a diameter of $8.2 \mathrm{~mm}$ and a mass of $660 \mathrm{mg}$ was placed into the container. $100 \mu \mathrm{L}$ of liquid medium (distilled water or PEG solution) were added to keep the hydrogel wet. Two spheroidal containers were placed in the friability tester and were subjected to 15 min rotation at 25 revolutions per min. After rotation the mass of the largest hydrogel fragment was measured and hydrogel mass loss $W_{\text {loss }}$ was calculated as

$$
W_{\text {loss }} \%=100 \%-\frac{W_{\max }}{W_{0}} \times 100 \%
$$

where $W_{\max }$ is the mass of the largest hydrogel fragment after rotation and $W_{0}$ is the initial mass of the hydrogel sample. Each experiment was repeated at least tree times and average value of the mass loss was taken.

\section{Scanning Electron Microscopy (SEM)}

The surface morphology of the hydrogels was examined using Carl Zeiss EVO HD15 Scanning Electron Microscope (UK) with the Peltier Coolstage MK3 (DEBEN UK Ltd) at various magnifications (up to 10K). Fully water-swollen gel samples were mounted on 12.5 SEM pin metal stubs and quickly frozen before images were collected. The temperature and pressure inside the sample chamber were $-21{ }^{\circ} \mathrm{C}$ at $10-15 \mathrm{~Pa}$, respectively.

\section{Calculation of the amount of freezing and non-freezing water in hydrogels}

The amount of non-freezable bound water $W_{\text {bound }}$ in total water was assessed by subtracting the amount of freezing water from the total water content using the equation

$$
W_{\text {bound }}(\%)=W_{\text {total }}(\%)-\left(\frac{\Delta H_{\text {freezing }}}{\Delta H_{\text {pure }}} \times 100 \%\right)
$$

where $W_{\text {total }}$ is the total water content in fully swollen hydrogel, $\Delta H_{\text {freezing }}$ is the melting enthalpy of freezable water (combined free and freezable bound water), $\Delta H_{\text {pure }}$ is the melting enthalpy of pure water (334 J g $\left.{ }^{-1}[17]\right) . \Delta H_{\text {freezing }}$ 
values were calculated from the area under the endothermic melting peak on DSC heating run. The total water content in hydrogels was calculated from the mass loss by hydrogels upon temperature-induced water evaporation measured by TG.

\section{Results}

\section{Swelling of HEA-HEMA hydrogels in water and in 20 vol\% PEG 400 (600) solutions.}

Hydrogels were fully swollen either in water or in $20 \mathrm{vol} \%$ PEG solutions with PEG average molecular weights of 400 or 600 Da. Fig. 1 (a) shows the relationship between the equilibrium swelling degree of HEA-HEMA hydrogels and the HEA content in hydrogels. As is seen, the amount of liquid in HEA-HEMA hydrogels in all solutions tested strongly depends on the copolymer composition and increases when the HEA content increases. The dependence on the HEA content is not linear; significant increase in the water uptake by the hydrogels occurs when the HEA content exceeded 40-50 mol\% (Fig. 1 (a)). Especially remarkable increase in the equilibrium swelling degree is observed in the case of HEA-rich hydrogels with the HEA content $\geq 70 \mathrm{~mol} \%$. Fig. 1 (a) also shows that the equilibrium swelling degrees of hydrogels depend on the liquid medium and decrease in more viscous media (20 vol\% PEG solutions).

Kinetics of hydrogel swelling (Fig. 1 (b)) shows that swelling of HEA-rich hydrogels (HEA $\geq 70 \mathrm{~mol} \%$ ) occurs faster than swelling of HEMA-rich hydrogels (HEA $\leq 40 \mathrm{~mol} \%)$ both in aqueous and in mixed solutions containing PEG 400 (600).

\section{Morphology of HEA-HEMA hydrogels in aqueous solution.}

The internal structure of water-swollen hydrogels was examined using SEM. The SEM micrographs of HEA-HEMA hydrogels at swelling equilibrium are shown in Fig. 2. All hydrogels have a porous structure with a low interconnectivity between individual pores in the case of PHEMA and highly interconnected pores in the case of HEA-rich hydrogels. PHEMA hydrogels show a very dense structure with the comparable linear sizes of pores and inter-pore polymeric "walls". As the fraction of HEA in the co-polymeric network increases, the hydrogels show a remarkable increase in porosity leading to a decrease in the polymer "wall" thickness and increase in (pore diameter):(polymer "wall" thickness) ratio. These structural changes result from the increased interaction between more hydrophilic HEA fragments and water molecules. It is supposed that the pores are the regions of mobile phase permeation. Comparison of the SEM images (Fig. 2) and the swelling degree dependence on the HEA content (Fig. 1 (a)) shows that the increased porosity of the HEA-rich hydrogels is observed in the hydrogel samples with the enhanced water sorption capability.

\section{Mechanical stability of HEA-HEMA hydrogels in aqueous and PEG solutions}

It is well known that the addition of PEG to aqueous solution increases the solution viscosity. Kinematic viscosities of $20 \mathrm{vol} \%$ PEG solutions used in the present work were found to be 2.58 and $3.02 \mathrm{mPa}$ s for PEG 400 and PEG 600 solutions, respectively. To test whether PEG presence in the liquid media could improve the hydrogel mechanical 
properties, friability of HEA-HEMA hydrogels with high HEA content (> $50 \mathrm{~mol} \%$ ) fully swollen in water or in 20 vol\% PEG 400 (600) mixed solutions was assessed.

Fig. 3 shows the mass loss of the HEA-rich hydrogel samples in water and in 20 vol\% solutions of PEG 400 and PEG 600 after the mechanical stress was applied. The mass loss values were calculated according to Eq. (2). As is seen, the mass loss increases with increased HEA content in hydrogels reflecting the fact that highly porous HEArich hydrogels containing a large amount of liquid are more fragile and easily brake into pieces. Hydrogels containing PEG keep their integrity much better and lose less mass under mechanical stress applied. PEG 600 (20 vol $\%$ solution) shows stronger effect on the hydrogel mechanical properties than 20 vol\% of PEG 400 . These results show that when PEG is introduced in the liquid medium it is able to significantly improve the mechanical properties of HEA-HEMA hydrogels with high HEA content making them more stable and less fragile.

\section{The total amount of water in HEA-HEMA hydrogels measured by TG}

The total amount of water is one of the main characteristics of hydrogels, since they are supposed to have an extraordinary capacity for imbibing water into the network structure. To assess the total amount of water in HEAHEMA hydrogels the TG was performed from 25 to $250{ }^{\circ} \mathrm{C}$ and the total mass loss of the samples was calculated. Fig. 4 presents the values of the total amount of water in HEA-HEMA hydrogels containing aqueous or 20 vol\% aqueous-PEG liquid phases. The water uptake by the hydrogels strongly depends on their chemical composition and remarkably increases with the HEA content both in water and in 20 vol\% PEG 400 (600) mixed solutions. Hydrogels fully equilibrated in $20 \mathrm{vol} \%$ PEG solutions contain less amount of water than those fully equilibrated in pure water (Fig. 4) with only a minute difference between the water content in hydrogels swollen in 20 vol\% mixed solutions containing PEG400 or PEG600.

\section{Water-retention properties of HEA-HEMA hydrogels in aqueous and PEG solutions studied by TG}

To study how the hydrogel composition and the presence of $20 \mathrm{vol} \%$ PEG 400 (600) in formulation affect the waterretention properties of hydrogels, a drying of swollen HEA-HEMA samples at physiological temperature $\left(37^{\circ} \mathrm{C}\right)$ was conducted using TG. Before experiments, the hydrogels were fully equilibrated either in water or in $20 \mathrm{vol} \%$ PEG solutions. In a typical 'loss-on-drying' experiment, each hydrogel sample was held during 120 min at $37{ }^{\circ} \mathrm{C}$ and constant purged gas flow and the sample mass loss over time was recorded. Typical 'loss-on-drying' TG curves for different hydrogels in 20 vol\% PEG 600 are presented in Fig. 5 (a). Mass of the hydrogel samples decreases over time due to water expelling from hydrogels.

Figure 5(b) shows the dependencies of the amount of water lost after two hours of drying at $37{ }^{\circ} \mathrm{C}$ as a function of the HEA content in HEA-HEMA hydrogels equilibrated in water or in 20\% PEG 400 (600) solutions. The results show that in the case of HEA-rich hydrogels, a negligible amount of residual water remained after the drying stage, evidencing that water is evaporated from HEA-rich hydrogels relatively easily. More water retains in HEMA-rich hydrogels (HEA < $40 \mathrm{~mol} \%$ ). The presence of $20 \mathrm{vol} \%$ PEG in formulations does not change the profiles of water evaporation. The character of water evaporation from hydrogels remains similar for hydrogels containing water or 20 vol\% PEG solutions as a liquid medium. Hydrogels equilibrated in 20 vol\% PEG 600 retain slightly more ( 3-5\%) 
water than hydrogels equilibrated in water (Fig. 5 (b)).

TG loss-on-drying curves schematically can be split into two stages: initial relatively fast loss of mass (i.e. fast water evaporation) followed by slow mass loss (i.e. slow water evaporation). To estimate the amount of fast and slowly evaporating water in HEA-HEMA hydrogels the inflection points were identified using Pyris TGA software (onset option). Fig. 5(c) shows the dependencies of the inflection times on the HEA content in hydrogels. As is seen, the inflection point is shifting towards longer times when the HEA content in hydrogels increases. The percentages of fast and slowly evaporating water calculated using the values of the sample masses at the inflection points are presented in Fig. 5(d).

\section{Study of the thermal properties of water in HEA-HEMA hydrogels by DSC.}

Fig. 6(a) presents typical DSC curves of HEA-HEMA hydrogels in 20 vol\% PEG 600 solution. All curves were obtained during the cooling run from 30 to $-45^{\circ} \mathrm{C}$ followed by a heating run from -45 to $30{ }^{\circ} \mathrm{C}$. For all samples studied (hydrogels swollen in water, in 20\% PEG400 (or 600) solutions; pure water and 20\% aqueous PEG solutions), two thermal events were observed: (i) sharp exothermic peak on the cooling stage related to crystallization of free water in the sample; (ii) broad endothermic peak on the heating stage due to the melting of water which was frozen during the cooling stage. The melting enthalpy values presented in Fig. 6 (b) increase when the HEA content increases indicating that the amount of freezable water strongly rises in HEA-rich hydrogel samples as they contain more water per unit of hydrogel mass. The dependencies in Fig. 6(b) are non-linear ("S"-shaped) indicating the significant changes in the HEA-HEMA hydrogel properties with a threshold near $40 \mathrm{~mol} \%$ of HEA and the maximum melting enthalpy values achieved for samples containing 80-100 mol\% of HEA. The absolute value of crystallization enthalpy also increases with the HEA content (data not shown).

\section{Estimation of the amount of free and bound water in HEA-HEMA hydrogels in aqueous and aqueous-PEG solutions.}

The quantities of non-freezing $\left(W_{\text {bound }}\right)$ and freezing water $\left(W_{\text {freezing }}\right)$ within the HEA-HEMA hydrogels were determined from the area under the endothermic peak in DSC curves (Fig. 6(a)) according to Eq. (3). The melting enthalpy of pure water measured by DSC in our experiments has been found to be equal to $335 \mathrm{~J} \mathrm{~g}^{-1}$ which is in very good agreement with the literature data $\left(334-340 \mathrm{~J} \mathrm{~g}^{-1}[17,18,26]\right)$. The total amount of water in hydrogels was measured by TG (Fig. 4). The quantities of freezing and non-freezing bound water are expressed as percentages of the total amount of water in fully swollen hydrogels.

Fig. 7 shows the amount of freezing water in HEA-HEMA hydrogels equilibrated in aqueous and in 20 vol $\%$ aqueous-PEG solutions. One can see that for water-swollen hydrogels the amount of freezing water significantly increases with HEA content from about 65\% in PHEMA to about 90-95\% in HEA90 and PHEA hydrogels. Consequently, the proportion of non-freezing water decreases with increased HEA content. 
As the content of liquid medium changes from pure water to $20 \mathrm{vol} \%$ water-PEG solution, the amount of freezing water in hydrogel samples decreases (Fig. 7). HEA-rich hydrogels show greater difference in the amount of freezing water between hydrogels equilibrated in water and in PEG solutions than HEMA-rich hydrogels.

\section{Discussion}

The equilibrium swelling degree of HEA-HEMA hydrogels strongly depends on HEA/HEMA ratio in the monomer mixture used for their synthesis. Increase in the HEA content above 40-50 mol\% results in a remarkable rise in the swelling degree from about 200\% for HEA40 up to about 1400\% for HEA90 (Fig. 1(a)). Comparison of the swelling degree and the total water content in hydrogels (Fig. 4) shows that above 80-85\% of the mass of the HEA70-HEA80 hydrogel samples is the liquid phase; and for HEA90-PHEA water-swollen hydrogels the water content is as high as 93-94 mass\%. At the same time PHEMA hydrogel contains only 55 mass\% of water. The difference in hydrogel swelling is related to the difference in the chemical structures of 2-hydroxyethyl methacrylate (HEMA) and 2hydroxyethyl acrylate (HEA) - lacking one methyl group, HEA is more hydrophilic than HEMA and can attract more water.

It is not surprising that a material containing $85-90$ mass\% of water shows weak mechanical properties. However, our results clearly show that the mechanical properties of HEA-HEMA hydrogels with high HEA content can be significantly improved by adding viscous co-solvent (PEG) to the hydrogel liquid medium. As is seen in Fig. 3, HEA-rich hydrogels containing 20 vol\% PEG can better withstand the mechanical stress and preserve their integrity during friability test than the hydrogels swollen in pure water. More viscous $20 \mathrm{vol} \%$ PEG with molecular weight of 600 (kinematic viscosity $3.02 \mathrm{mPa} \mathrm{s}$ ) protects hydrogels from the mechanical stress better than $20 \mathrm{vol} \%$ PEG400 (kinematic viscosity $2.58 \mathrm{mPa} \mathrm{s}$ ).

In mixed solutions containing 20 vol\% PEG the equilibrium swelling degree of HEA-HEMA hydrogels decreases but still remains high (above 900-1000\% for HEA-rich hydrogels with HEA > 70 mol\%) compared to the swelling degree of PHEMA (below 200\%) (Fig. 1(a)).

Similar to the equilibrium swelling degree, the rate of hydrogel swelling also decreases in 20 vol\% PEG solutions. To determine the mechanism of solvent diffusion into the polymer matrix the following power law equation $[1,27]$ can be used:

$$
\frac{M_{t}}{M_{0}}=k t^{n}
$$

where $\mathrm{M}_{\mathrm{t}}$ and $\mathrm{M}_{0}$ are the amount of water absorbed by the hydrogel at time $t$ and at equilibrium, $k$ is a characteristic constant of the system and $n$ is a characteristic exponent of the mode of water transport. Equation (4) is applicable only to the initial stage of swelling when $\mathrm{M}_{\mathrm{t}} / \mathrm{M}_{0} \leq 0.6$. Equation (4) can be re-written as 
The swelling kinetics data were re-plotted in logarithmic coordinates $\ln \left(\mathrm{M}_{\mathrm{t}} / \mathrm{M}_{0}\right)$ vs. $\ln (\mathrm{t})$. The intercept and slope of the linear curves obtained were used to calculate the values of the kinetic constant $k$ and diffusion exponent $n$. Values of diffusion exponent $n$ calculated for HEA-HEMA hydrogel swelling in water [24] and solutions containing 20 vol\% PEG 400 (600) are shown in Fig. 1(c).

Depending on the rates of liquid diffusion into the polymer matrix and polymer relaxation, three types of diffusion mechanism can be considered [1, 28, 29]. For the Fickian diffusion (Case 1) with $n=0.5$, the rate of water diffusion is lower than the rate of relaxation of the polymer chain making the diffusion a rate limited factor. Coefficient $n=1$ indicates Case 2 diffusion where the rate of diffusion is rapid compared with the chain relaxation process. NonFickian diffusion or Case 3 diffusion $(0.50<n<1)$ occurs when the diffusion and relaxation rates are comparable and hydrogel swelling is controlled by both liquid phase diffusion inside the polymer matrix and by relaxation of polymer chains $[1,27]$.

Analysis of the results (Fig. 1 (c)) shows that for hydrogels swollen in pure water nearly linear dependence of $n$ on HEA content occurs. HEMA-rich samples (HEA < 60) revealed Fickian type of diffusion of water into hydrogels $(n$ in the range of $0.33-0.45)$. At the same time HEA-rich hydrogels show non-Fickian type of diffusion $(n>0.5)$. Similar observations were reported for HEMA-based hydrogels in [30, 31] with $n$ equal to 0.44 for PHEMA and 0.50.53 for HEMA with more hydrophilic copolymers. In 20 vol\% PEG solution swelling of HEMA-rich hydrogels (HEA $<70$ mol\%) becomes "less Fickian" [32] with $n<0.5$ (Fig. 1 (c)), meaning that increased viscosity of the liquid medium containing PEG makes its diffusion into the polymer matrix very slow, thus limiting the rate of the swelling process. In this case the water penetration rate is much below the polymer chain relaxation rate. HEA-rich samples (HEA > $80 \mathrm{~mol} \%$ ) show non-Fickian diffusion $(0.5<n<1)$ in 20 vol\% PEG solutions as well as in water (Fig. 1 (c)).

One of the main properties of hydrogels is their ability not only to imbibe water but also to retain it making possible the sustained release of the loaded medicines. TG 'loss-on-drying' experiments allow a convenient way to study water evaporation from hydrogels at different temperatures. The TG results (Fig. 5(b)) show that the rate of water evaporation from hydrogels at physiological temperature $\left(37^{\circ} \mathrm{C}\right)$ strongly depends on the hydrogel composition after 2 hours' incubation HEMA-rich hydrogels lose significantly less water (up to 15-17\%) than HEA-rich hydrogels due to their denser structure preventing water from escape. PEG400 has been shown to have an insignificant effect on the amount of water evaporated from HEA-rich (HEA $\geq 70$ mol\%) hydrogels, however its presence helps HEMA-rich hydrogels (HEA $\leq 40$ vol\%) to retain water better (Fig.5 (b)). Both HEA-rich and HEMA-rich hydrogels containing 20 vol\% PEG600 in the liquid medium retained more water after $120 \mathrm{~min}$ incubation at physiological temperature; however, as the results show, the overall increase in water retained is only $3-5 \%$ of the total amount. 

HEMA hydrogels the water evaporation profiles consist of "fast" and "slow" evaporation stages. It can be assumed that water evaporating during the "fast" stage is easily accessible and has little or no interaction with the polymer matrix (bulk water). On the contrary, "slowly" evaporating water requires more time to escape from hydrogel, probably, due to its H-bonding to the polymer matrix or to bound water. The more free water hydrogel contains, the longer the "fast" evaporating stage is expected to carry on and the more percentage of water is expected to be expelled during the fast stage.

The duration of the "fast" evaporation stage clearly depends on the HEA content and increases from about 30 min for PHEMA to about 60 min for HEA-rich hydrogels (Fig. 5(c)). At the same time the amount of "fast" evaporating water also increases with increased HEA content - about $85 \%$ of the total water is evaporated during the fast stage from PHEMA and HEMA-rich hydrogels (HEA < $40 \mathrm{~mol} \%$ ) and almost 97-100\% of water from HEA-rich hydrogels (HEA $\geq 80 \mathrm{~mol} \%$ ). The presence of $20 \mathrm{vol} \%$ PEG in liquid medium does not change the profiles of water evaporation from hydrogels. PEG400 has only a minor effect on the inflection time and the amount of fastevaporating water. The presence of PEG600 in liquid medium slightly increases the duration of the fast stage and decreases the amount of fast-evaporating water (Fig. 5(c and d)).

Overall, the results show that the hydrogels equilibrated in 20 vol\% PEG 600 retain more water than the samples swollen in water, however, the difference is rather small. One can conclude that the water-retention properties of HEA-HEMA hydrogels swollen in water and in PEG solutions strongly depend on the composition of the polymer network and, to a lesser extent, depend on the composition of the swelling liquid medium.

Combination of TG and DSC thermoanalytical methods also allows assessing the amount of freezing (bulk and intermediate) and non-freezing (bound) water in hydrogels. The crystallization peak on the DSC curves of all samples (Fig. 6 (a)) indicates the presence of freezable water in hydrogels equilibrated in aqueous and in PEG solutions. For partly dried hydrogel samples the area under the crystallization peak significantly decreased and eventually peak disappeared. For example, for the HEA10 hydrogel the crystallization peak disappeared when the water content in hydrogel was reduced from $\sim 60$ (for hydrogel fully equilibrated in water) to $\sim 38$ mass $\%$ (data not shown). Therefore certain minimum water content in hydrogels is required for free water to appear.

The enthalpies of crystallization and melting measured by DSC were used to assess the amount of freezing water in hydrogels. Fig. 7 shows that the amount of freezable water in water-swollen hydrogels increases with increased HEA content. PHEMA hydrogel with the lowest water content possesses the smallest free-to-bound water ratio. As the HEA content in hydrogel increases, the total amount of water and the free-to-bound water ratio increase significantly. In the case of HEA-rich hydrogels the amount of freezable water rises due to a substantial increase in porosity of the HEA-rich hydrogels. It is expected that more hydrophilic HEA polymer contains a greater proportion of bound water. At the same time addition of HEA to HEMA within the copolymers leads to a polymer network with a lower crosslinking density (larger mesh size) and greater interconnecting pores accumulating more free water (Fig. 2). Perhaps, these two processes are in equilibrium in the case of intermediate HEA contents (30-60 mol\%) where the swelling 
degree is moderately high (Fig. 1(a)) and the amount of freezable water in hydrogels does not change significantly with increased HEA content (Fig. 7).

In aqueous PEG solutions, a part of water molecules forms H-bonds with hydrophilic molecules of PEG increasing the overall amount of bound water. The latter results in a decrease in the melting enthalpies $\left(\Delta \mathrm{H}_{\text {melt }}\right)$ of the mixed solutions: $\Delta \mathrm{H}_{\text {melt }}$ for 20 vol\% PEG600 and PEG400 were 168 and 194, respectively, indicating that about 42 and $50 \%$ of all water in mixed solutions becomes non-freezable. When 20 vol\% PEGs (400 or 600) are added to the hydrogel liquid medium, the total amount of bound water is also expected to grow thus lowering the amount of free water (Fig. 7).

Overall, the results obtained show that the addition of the viscous co-solvent (PEG) to the liquid medium results in significant improvement of the mechanical properties of HEA-HEMA hydrogels and also slightly retards the water loss from hydrogels. A redistribution of free and bound water in the hydrogel samples occurring in mixed solutions containing $20 \mathrm{vol} \%$ PEG can have an impact on the rate of drug diffusion to/from hydrogels.

\section{Acknowledgements}

The authors would like to thank Rachel Armitage for help with recording SEM images. G.M.B. Tiguman acknowledges the "Science Without Boarders" student exchange program and the University of San Paulo, Brazil.

\section{References}

1. Peppas NA, Bures P., Leobandung W., Ichikawa H. Hydrogels in Pharmaceutical Formulations. Eur J Pharm Biopharm. 2000;50:27-46.

2. Lin CC., Metters AT. Hydrogels in controlled release formulations: network design and mathematical modeling. Adv Drug Deliv Rev. 2006; 58(12-13):1379-408.

3. Kim JK., Kim HJ., Chung JY., Lee JH., Young SB., Kim YH. Natural and synthetic biomaterials for controlled drug delivery. Arch Pharm Res. 2014;1:60-8.

4. Amin S., Rajabnezhad S., Kohli K. Hydrogels as Potential Drug Delivery Systems. Scientif Res Essay. 2011; 11:1175-83.

5 Hu X., Hao L., Wang H., et al. Hydrogel contact lens for extended delivery of ophthalmic drugs. Intl J Pol Sci. 2011;1-9.

6. Ribeiro A., Veiga F., Santos D., Torres-Labandeira JJ., Concheiro A., Alvarez-Lorenzo C. Bioinspired imprinted PHEMA-hydrogels for ocular delivery of carbonic anhydrase inhibitor drugs. Biomacromolecules. 2011;12(3):701-9.

7. Cetin D., Kahraman AS., Gümüşderelioğlu M. Novel scaffolds based on poly(2-hydroxyethyl methacrylate) superporous hydrogels for bone tissue engineering. J Biomater Sci Polym Ed. 2011;22:1157-78. 
8. Slaughter B., Khurshid S., Fisher OZ., Khademhosseini A., Peppas NA. Hydrogels in Regenerative Madicine. Adv Mater. 2009;21:3307-29.

9. John MS., Andrade JD. Water and hydrogels. J Biomed Mater Res. 1973; 7:509-15.

10. Salmerón-Sánchez M., Monleón Pradas M., Gómez Ribelles JL. Thermal transitions in PHEA hydrogels by thermomechanical analysis. A comparison with DSC data. Europ Pol J. 2004; 40(2):329-34.

11. Wood JM., Attwood D., Collett JH. The influence of gel formulation on the diffusion of salicylic acid in polyHEMA hydrogels. J Pharm Pharmacol. 1982; 34(1):1-4.

12. Wood JM., Attwood D., Collett JM. Characterization of poly(2-hydroxyethylmethacrylate) gels. Drug Devel Ind Pharm. 1983;9(1-2):93-101.

13. Mackie JS., Meares P. The diffusion of electrolytes in a cation exchange resin membrane. Proceedings of the Royal Society of London. 1955;232:498-509.

14. McBrierty VJ., Martin SJ., Karasz FE. Understanding hydrated polymers: the perspective of NMR. J Mol Liquids. 1999;80:179-205.

15. Sakai Y., Kuroki S., Satoh M. Water properties in the super-salt-resistive gel probed by NMR and DSC. Langmuir. 2008;24(13):6981-7.

16. Baumgartner S., Lahajnar G., Sepe A., Kristl J. Investigation of the state and dynamics of water in hydrogels of cellulose ethers by 1H NMR spectroscopy. AAPS PharmSciTech. 2002;3(4):E36.

17. Bouwstra JA., Salomon-de Vries MA., van Miltenburg JC. The thermal behaviour of water in hydrogels. Thermochimica Acta 1995;248:319-27.

18. Goda T., Watanabe J., Takai M., IshiharaK. Water structure and improved mechanical properties of phospholipid polymer hydrogel with phosphorylcholine centered intermolecular cross-linker. Polymer;4:139096.

19. Tomar N., Tomar M., Gulati N., Nagaich U. pHEMA hydrogels: Devices for ocular drug delivery. Int J Health and Allied Sci. 2012;4:224-30.

20. Paterson SM., Shadforth AM., Shaw JA., Brown DH., Chirila TV., Baker MV. Improving the cellular invasion into PHEMA sponges by incorporation of the RGD peptide ligand: the use of copolymerization as a means to functionalize PHEMA sponges. Mater Sci Eng C Mater Biol Appl. 2013;33(8):4917-22.

21. Dursch TJ., Taylor NO., Liu DE., Wu RY., Prausnitz JM., Radke CJ. Water-soluble drug partitioning and adsorption in HEMA/MAA hydrogels. Biomaterials. 2014;35(2):620-9.

22. Jovašević JS. , Dimitrijević SI., Filipović JM., Tomić SLj., Micić M.M. Swelling, Mechanical and Antimicrobial Studies of Ag/P(HEMA/IA)/PVP Semi-IPN Hybrid Hydrogels. Acta Physica Polonica a. 2011;2:279-83.

23. Hong KH., Jeon YS., Kim JH. Preparation and properties of modified PHEMA hydrogels containing thermoresponsive pluronic component. Macromol Res. 2009;1:26-30.

24. Khutoryanskaya OV., Mayeva ZA., Mun GA., Khutoryanskiy VV. Designing temperature-responsive biocompatible copolymers and hydrogels based on 2-hydroxyethyl(meth)acrylates. Biomacromolecules. 2008;9(12):3353-61.

25. Henning T. Polyethylene glycols (PEGs) and the pharmaceutical industry. PharmaChem. 2002;6:57-59. 
26. Ostrowska-Czubenko J., Gierszewska-Druzyńska M. Effect of Ionic Crosslinking on the Water State in Hydrogel Chitosan Membranes. Carbohydrate Polym. 2009;3:590-98.

27. Ritger PL., Peppas NA. A simple equation for description of solute release. I. Fickian and non-Fickian release from non-swellable devices in the form of slabs, spheres, cylinders or discs. J Controlled Release. 1987;5:23-36.

28. Katime I., Mendizábal E. Swelling properties of new hydrogels based on the dimethyl amino ethyl acrylate methyl chloride quaternary salt with acrylic acid and 2-methylene butane-1,4-dioic acid monomers in aqueous solutions. Materials Sci\&d Applicat. 2010;1:162-67.

29 De Kee D., Liu Q., Hinestroza J. Viscoelastic (Non-Fickian) diffusion. Canad J of Chem Engin. 2005;6:913-29.

30. George KA., Wentrup-Byrne E., Hill DJT., Whittaker AK. Investigation into the diffusion of water into HEMA-co-MOEP hydrogels. Biomacromolecules. 2004;5(4):1194-99.

31. Tomić S., Mićić M., Filipović J., Suljovrujić E. Swelling and drug release behavior of poly(2-hydroxyethyl methacrylate/itaconic acid) copolymeric hydrogels obtained by gamma irradiation. Radiat Phys\&Chem. 2007;5:801-10.

32. Wang J., Wu W., Lin Z. Kinetics and thermodynamics of the water sorption of 2-hydroxyethyl methacrylate/styrene copolymer hydrogels. J Appl Polym Sci. 2008;109:3018-23.

\section{Figure Captions:}

Fig. 1 HEA-HEMA hydrogel swelling in aqueous and mixed solutions containing 20 vol\% PEG400 or PEG600

(a) The equilibrium swelling degree of HEA-HEMA hydrogels as a function of the HEA co-polymer content for hydrogels fully swollen in water $(\Delta), 20$ vol\% PEG $400(\bullet)$ and 20 vol\% PEG $600(\bullet)$

(b) Typical swelling kinetics of HEA-HEMA hydrogels in 20 vol\% PEG 600 solution; HEA content, mol\%: 0 (•), $60(\Delta), 70(\mathbf{\square})$

(c) Effect of the HEA content on the diffusion exponent $n$ for hydrogels in water (०) [24], 20 vol\% PEG400 (•), 20 vol\% PEG600 ( $\boldsymbol{\Delta})$

Fig. 2 SEM micrographs (x5000) of the porous structure of HEA-HEMA hydrogels equilibrated in distilled water. HEA content, mol\%: (a) 0, (b) 10, (c) 50, (d) 80

Fig. 3 The mass loss after the mechanical stress for HEA-HEMA hydrogels equilibrated in water $(\Delta), 20$ vol\% PEG $400($ (•) or 20 vol\% PEG $600(\bullet)$ solutions 
Fig .4 Total amount of water in hydrogels with different HEA content measured by TG. Hydrogels were fully equilibrated in water $(\Delta), 20$ vol\% PEG $400(\Delta)$ or 20 vol\% PEG $600(\bullet)$ solutions. Solid lines represent fitting of the experimental data to sigmoidal (Boltzmann) function

Fig. 5 Water-retention properties of HEA-HEMA hydrogels studied by TG

(a) Typical loss-on-drying TG curves illustrating change in hydrogel mass over time for HEA-HEMA hydrogels in 20 vol\% PEG400 incubated at a constant temperature $37{ }^{\circ} \mathrm{C}$ during $120 \mathrm{~min}$. After 120 min hydrogels were heated up to $250^{\circ} \mathrm{C}$. HEA content (mol\%) from top to bottom: 0, 30, 40, 50, 70, 100. The dashed lines interception shows the inflection point (illustrated for HEA100\% only)

(b) Amount of water (\% of the total water) evaporated during $120 \mathrm{~min}$ incubation of hydrogels at $37{ }^{\circ} \mathrm{C}$ for hydrogels with different HEA content equilibrated in water $(\Delta), 20$ vol\% PEG400 (•), 20 vol\% PEG600 (•)

(c) Dependencies of the inflection time on HEA content for hydrogels in water $(\Delta), 20 \mathrm{vol} \%$ PEG400 ( $)$ and 20 vol\% PEG600 (•)

(d) The amount of water evaporated during the first (fast) stage vs. HEA content for hydrogels equilibrated in water $(\Delta), 20$ vol\% PEG400 (•), 20 vol\% PEG600 (•)

Solid lines (in Fig b, c, d) represent fitting of the experimental data to sigmoidal (Boltzmann) function

Fig. 6 Freezing and melting of water in HEA-HEMA hydrogels studied by DSC

(a) Typical DSC curves representing the freezing and melting curves for hydrogels in 20 vol\% PEG 600 solution. HEA content (mol\%) from top to bottom: 100, 90, 60, 40, 20, 10

(b) Enthalpies of melting $\Delta \mathrm{H}$ for freezable water in hydrogels with different HEA content fully equilibrated in water $(\Delta), 20$ vol\% PEG400 (•) or 20 vol\% PEG600 (•)

Fig. 7 Amount of freezing water $(\%)$ in HEA-HEMA hydrogels with different HEA content equilibrated in water $(\Delta)$, 20 vol\% solutions of PEG400 (•) or PEG600 (•) 


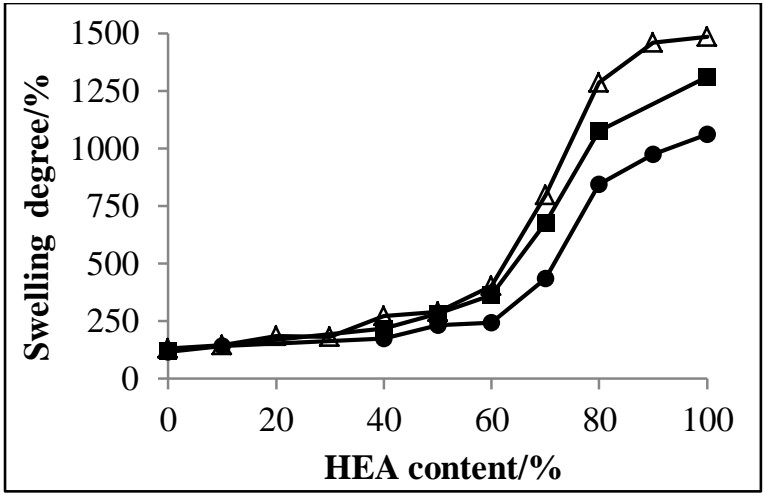

a

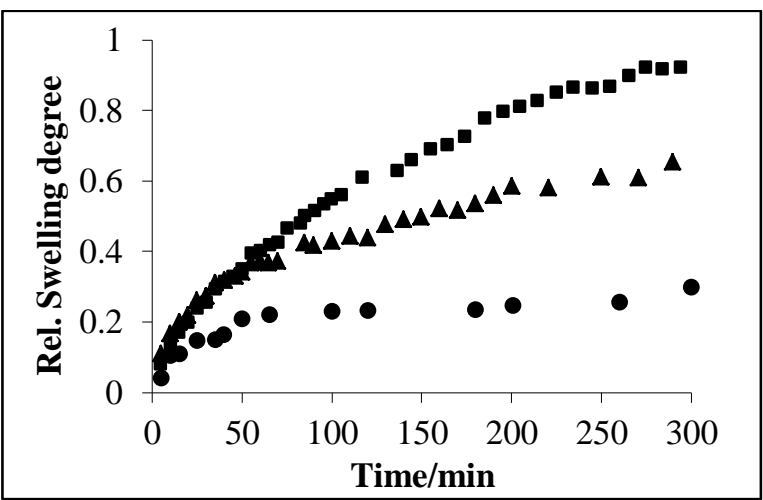

b

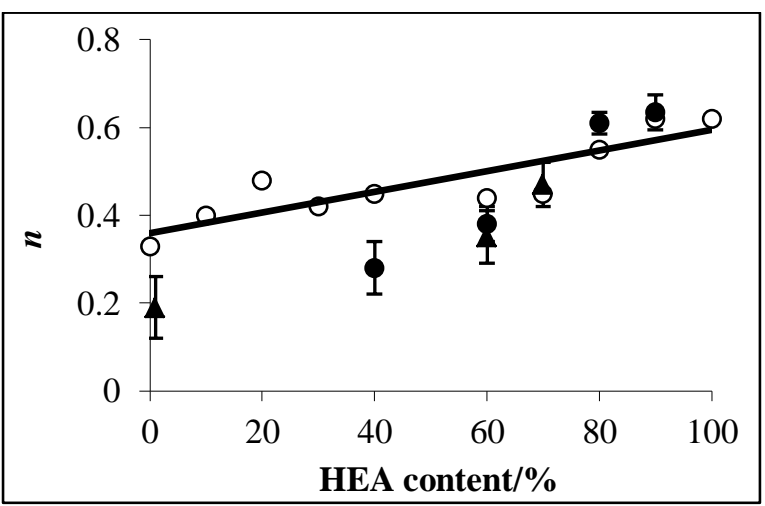

c

Fig. 1 

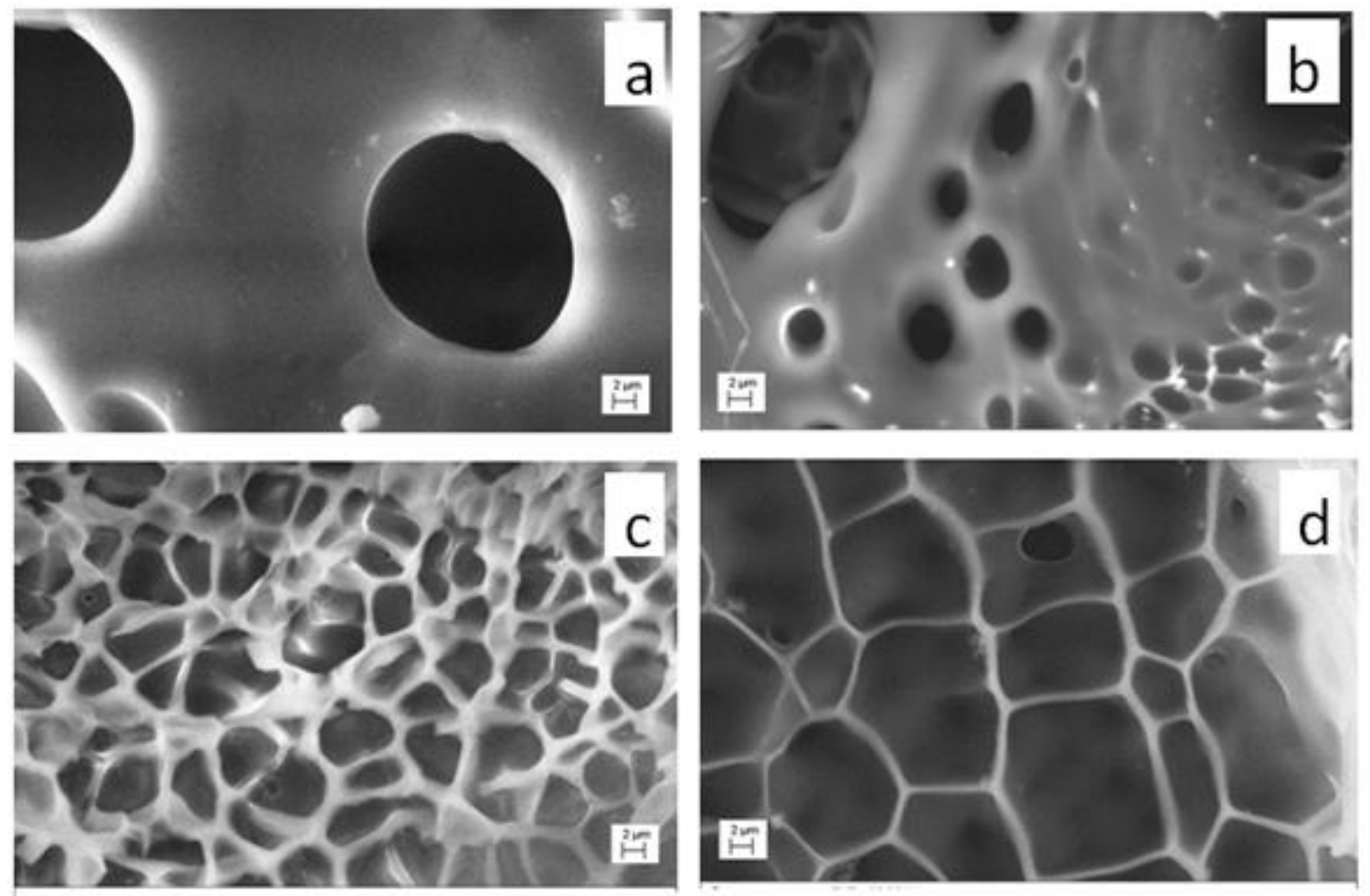

Fig. 2 


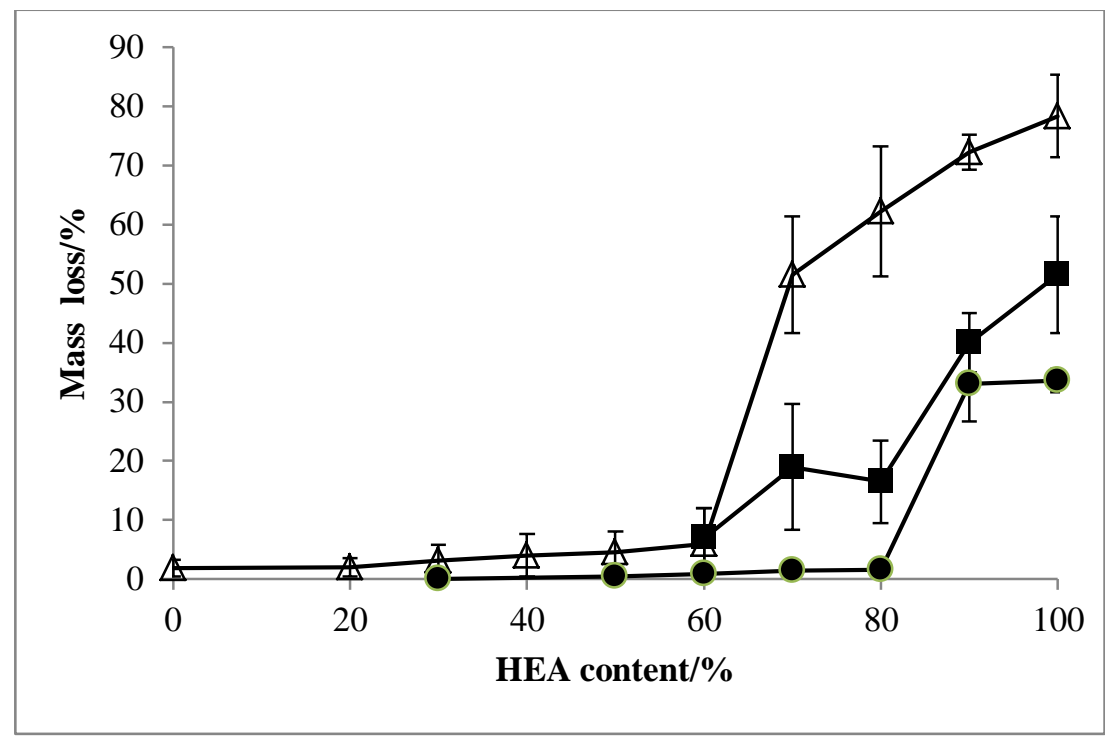

Fig. 3

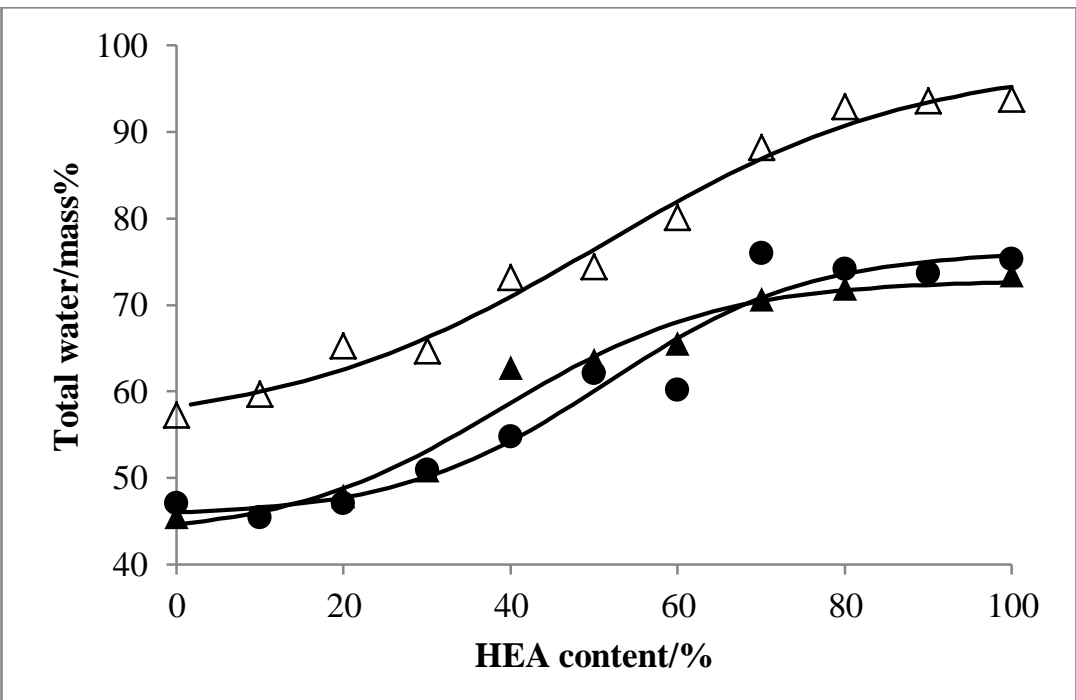

Fig. 4 


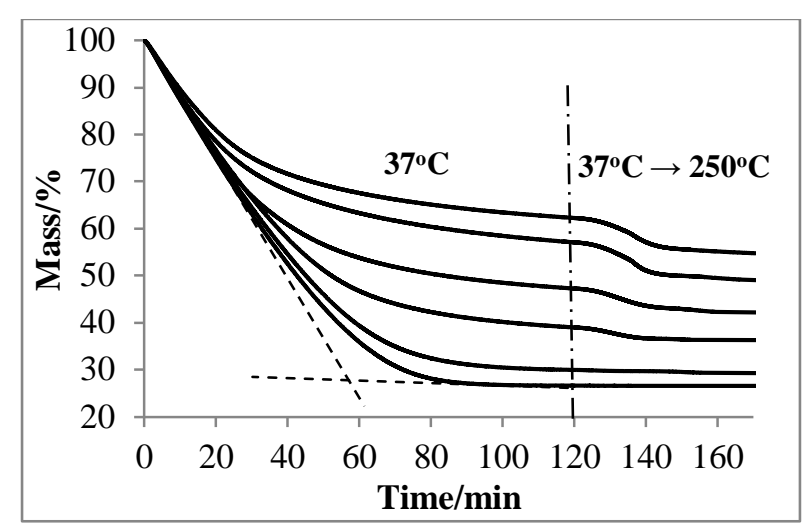

a

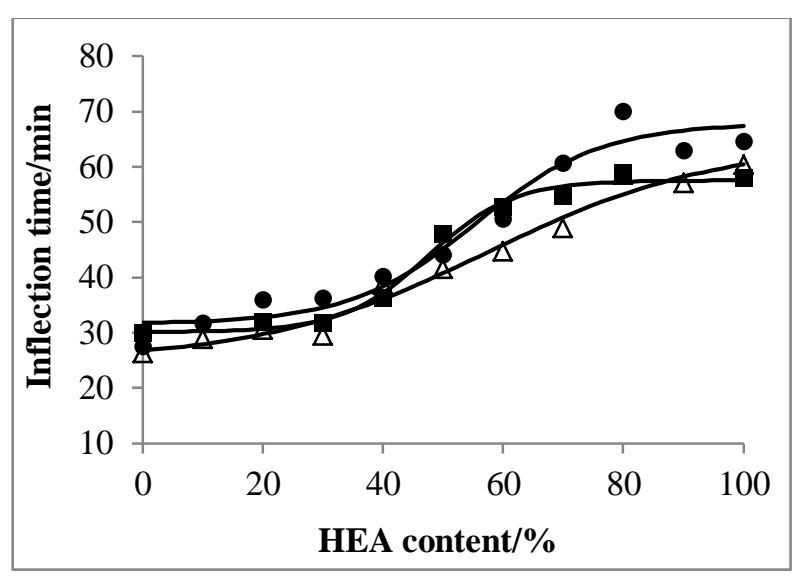

c

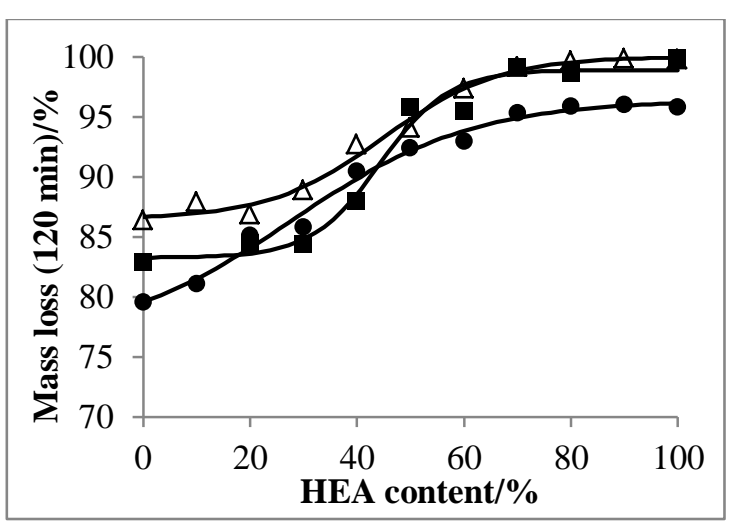

b

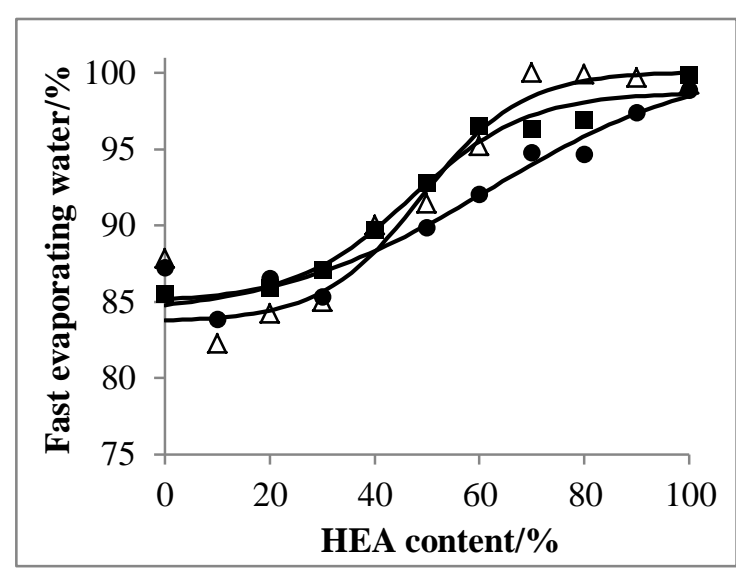

d

Fig. 5 


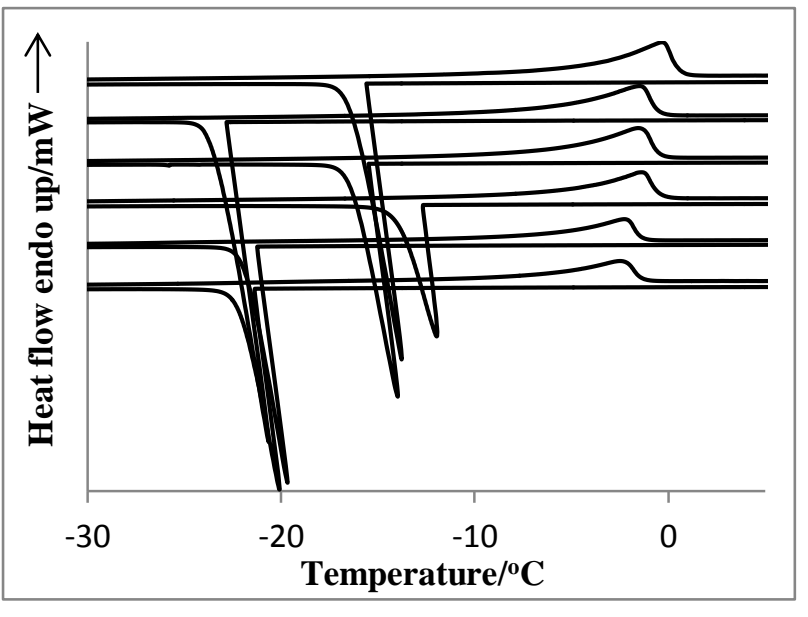

a

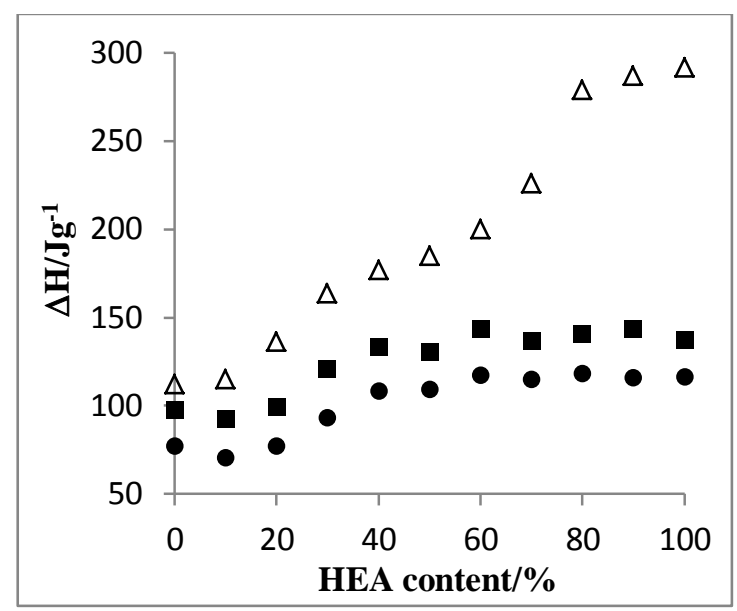

b

Fig. 6

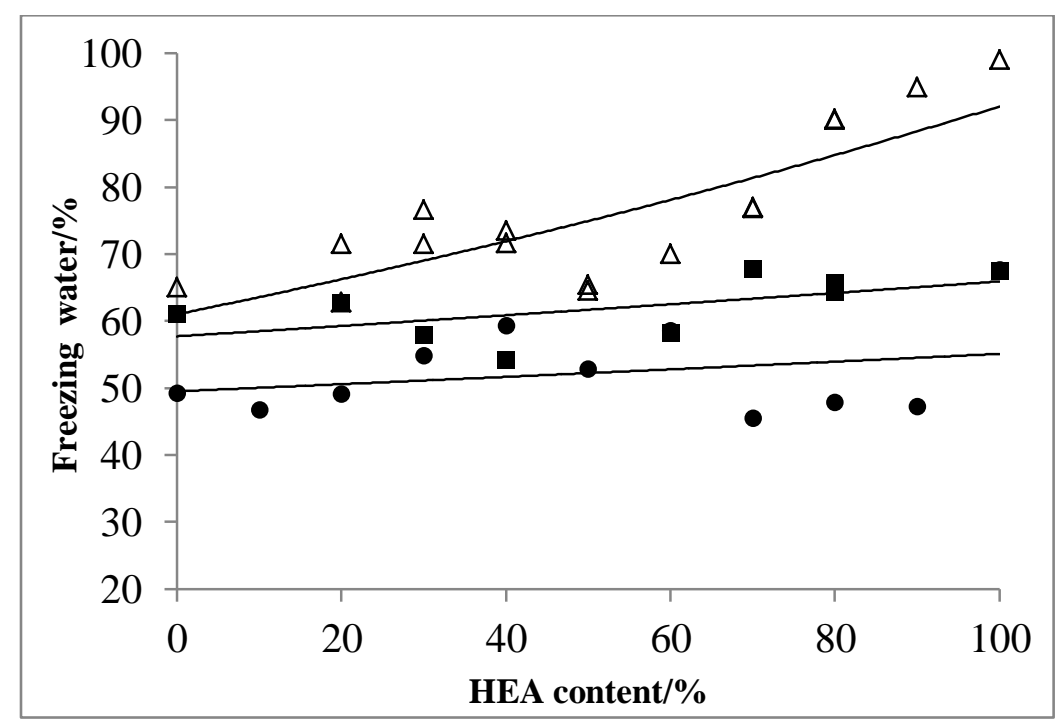

Fig. 7 


\section{Copyright Transfer Statement \\ Publishor: Akadbmiai Klado Zrt, Budapest, Hungary}

The signed Copprighn Transter Statement plesis rabuin se:

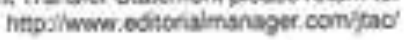

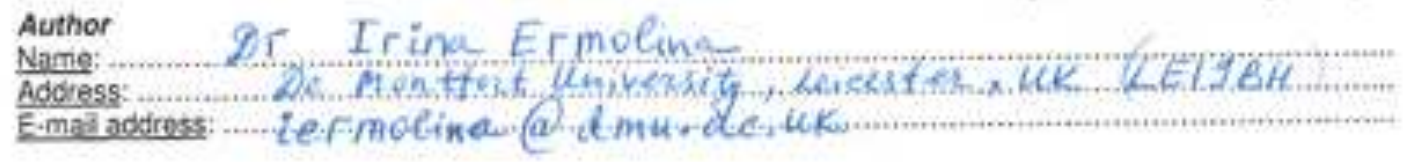

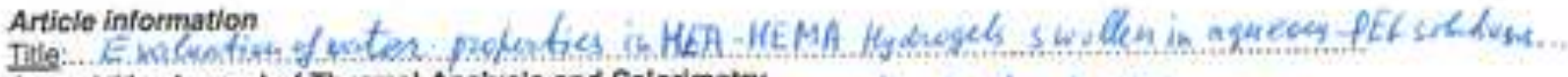
Soutnal lifie: Journal of Thermal Analysis and Calorimetry

1. Thanalee of ecerright

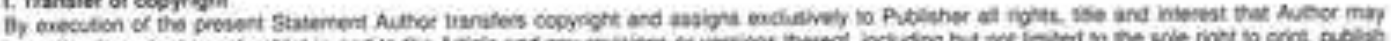

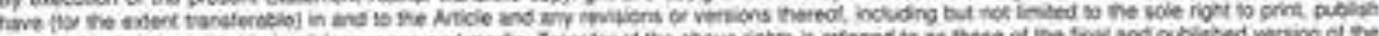

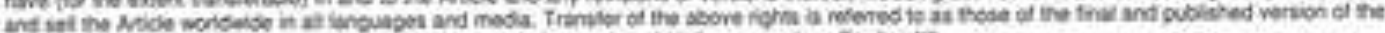

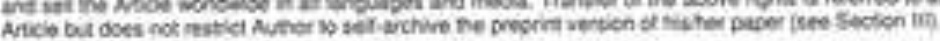

i. Rigta and obilgatiens of Publisher

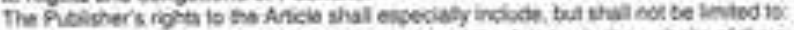

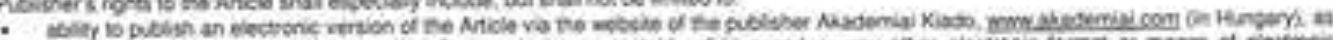

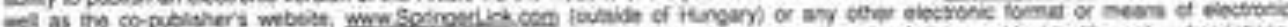

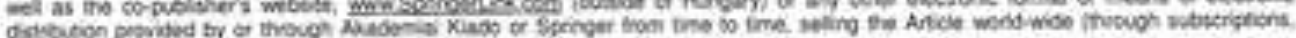
Par periview, single archive sale, exc)

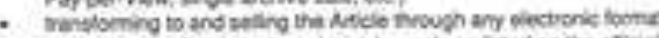

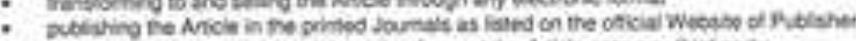

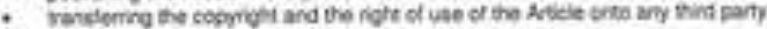

t. varsinging the Artolo

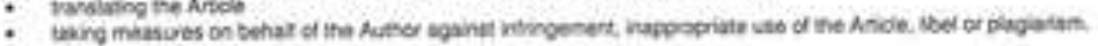

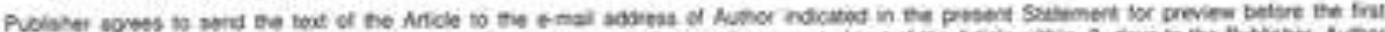

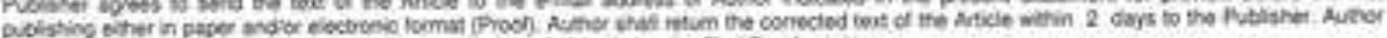

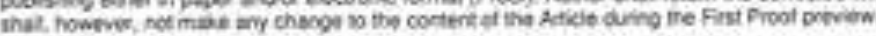

ai. Rephts and obliensons of Auther

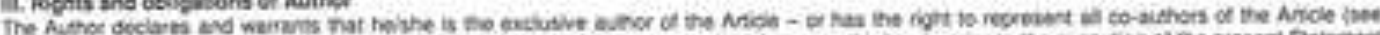

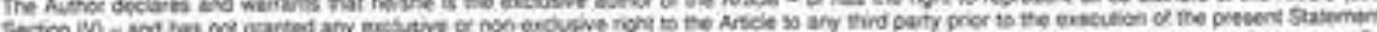
Stcton

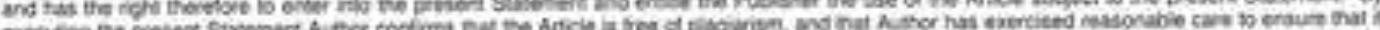

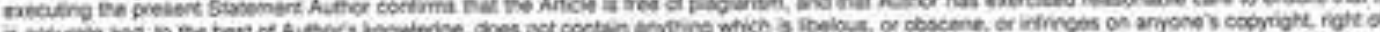

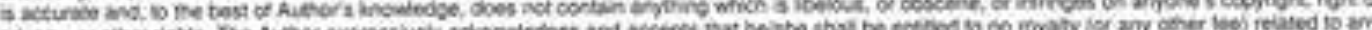

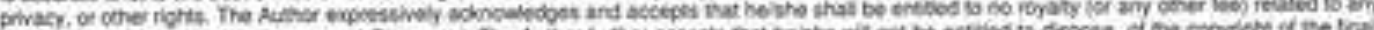

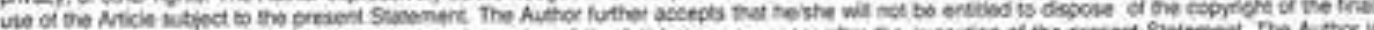

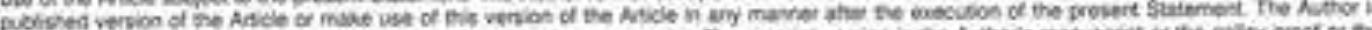

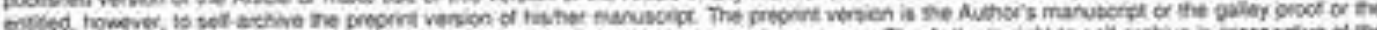

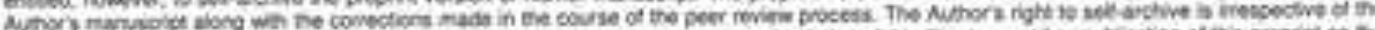

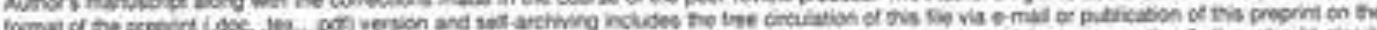

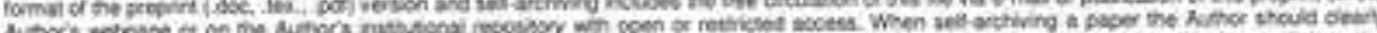

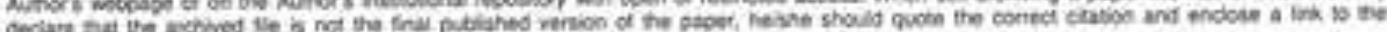

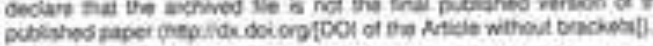

W. Uas of third party content as part of the Anticle

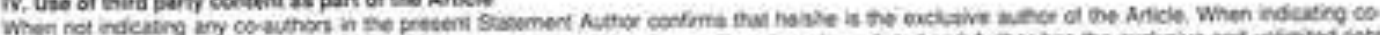

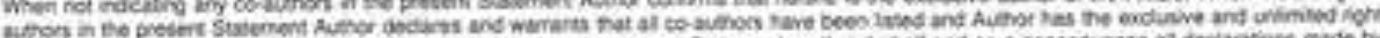

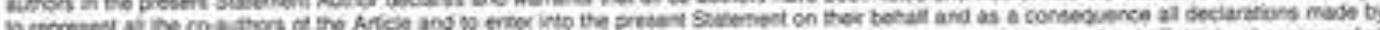

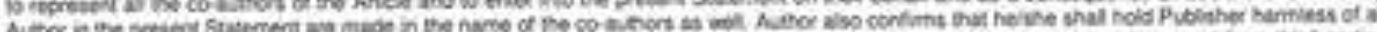

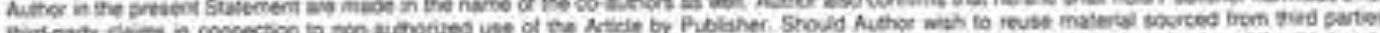

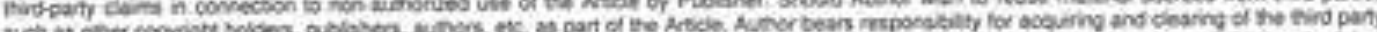

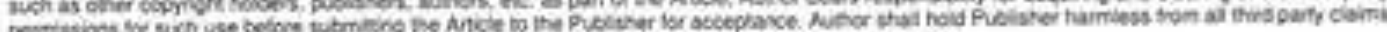

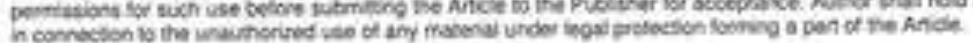

V. Oover provisions

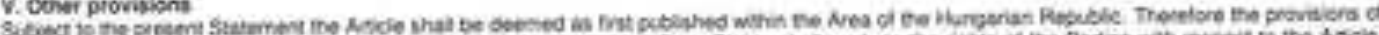

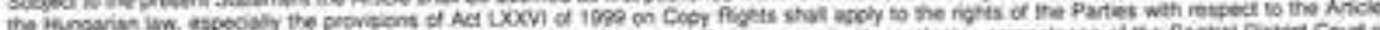

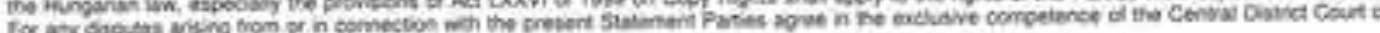

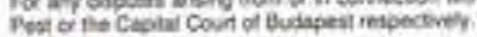

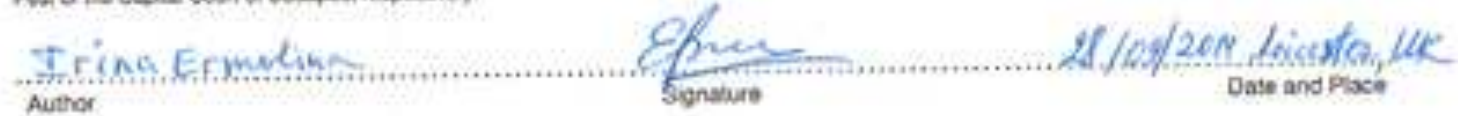

Author 